\title{
Percepções sobre homeopatia na perspectiva de discentes dos cursos de saúde de um centro universitário de Recife-PE
}

\author{
Ana Laura Pereira da Silva \\ Farmacêutica, Especialista em Farmácia Magistral \\ Centro Universitário Maurício de Nassau - UNINASSAU - Brasil \\ 凹analaura.farma@gmail.com \\ Jennifer Hellen Melo Sobral \\ Estudante de Farmácia \\ Centro Universitário Maurício de Nassau - UNINASSAU - Brasil \\ $\triangle$ melojennifer170@gmail.com \\ Juliana Marina Campelo \\ Estudante de Farmácia \\ Centro Universitário Maurício de Nassau - UNINASSAU - Brasil \\ $\triangle$ julyana_campelo@hotmail.com \\ Heytor Victor Pereira da Costa Neco \\ Biólogo, Mestre e Doutor em Ciências \\ Especialista em Metodologias Ativas \\ Centro Universitário Maurício de Nassau - UNINASSAU - Brasil \\ 凹hytorvictor@hotmail.com
}

Recebido em 6 de setembro de 2020

Aceito em 6 de julho de 2021

\begin{abstract}
Resumo:
A homeopatia é um método terapêutico que tem como princípio "semelhante cura semelhante", tendo como base a experimentação das preparações altamente diluídas e sucussionadas, com objetivo de produzir em pessoas sadias sintomas semelhantes aos da doença que devem combater. $O$ interesse da população por ela vem aumentando e há necessidade de formar profissionais qualificados no ensino de práticas integrativas e complementares para que avancem no SUS. Diante disso, o objetivo deste estudo foi descrever as percepções sobre homeopatia na perspectiva de discentes dos cursos de saúde de um centro universitário privado em Recife-PE. Para tanto, foi realizado um estudo transversal, descritivo e quantitativo a partir da aplicação de questionário a 500 estudantes de cursos de saúde, entre outubro e novembro de 2018. Apesar de $65,2 \%$ dos discentes relatarem ter ouvido falar sobre homeopatia, $73 \%$ declararam ter pouco ou nenhum conhecimento sobre as formas de tratamento. Além disso, 82,8\% dos discentes afirmaram não conhecer as PNPIC (Política Nacional de Práticas Integrativas e Complementares no SUS), e nem saber que medicamentos homeopáticos são distribuídos gratuitamente pelo SUS (86\%). Existe interesse dos estudantes de saúde em incluir homeopatia como disciplina na grade de seus cursos, afim de estarem aptos para oferecer um atendimento integral aos pacientes. Portanto, há necessidade de instituições de ensino superior investirem e promoverem disciplinas que abordem a homeopatia, para formarem profissionais com maior conhecimento sobre as PIC, o que irá acarretar maior informação para a população, contribuindo na melhoria da saúde individual e coletiva através da saúde pública.

Palavras-chave: Educação superior, homeopatia, terapias alternativas, SUS.
\end{abstract}




\title{
Healthcare students perceptions about homeopathy at a university of Recife-PE
}

\begin{abstract}
:
Homeopathy is a type of therapy method that follows the principle of "like cures like", based on the experimentation of high diluted preparations, with the aim of of producing in a healthy person, symptoms most 'like' the symptoms the person is suffering from. There has been increasing interest in these therapies and, therefore, is important to train qualified professionals focused on homeopathy and complementary and alternative medicine for work in the SUS. The aim of this study was to describe the perceptions about homeopathy from the perspective of healthcare students at a private university in Recife-PE. A cross-sectional, descriptive and quantitative study was conducted, between the months of October and November 2018. To assess opinions about homeopathy, 500 students were asked questions in a questionnaire. Although $65.2 \%$ of the students report having heard/read about homeopathy, 73\% have little or no knowledge about this alternative therapie. In addition, $82.8 \%$ did not know the PNPIC (National Policy for Integrative and Complementary Practices in the SUS), nor that homeopathic medicines are distributed free of charge by the SUS (86\%). Healthcare students are interested in including homeopathy as a discipline in their graduations, in order to be able to offer comprehensive care to patients. Therefore, there is a need for universities to invest and promote disciplines that address homeopathy, to train professionals with greater knowledge about alternative and complementary medicine, which will lead to more information for the population, contributing to the improvement of individual and collective health through public health.
\end{abstract}

Keywords: Higher education, homeopathy, alternative therapies, SUS.

\section{Percepciones sobre la homeopatía desde la perspectiva de discentes de cursos de salud de un centro universitario en Recife-PE}

\begin{abstract}
Resumen:
Homeopatía es un método terapéutico que tiene el principio de "cura similar similar", basado en la experimentación de preparados altamente diluidos, con objetivo de producir en una persona sana síntomas similares a los de la enfermedad que debe combatir. El interés por estas terapias ha ido en aumento y, por tanto, la necesidad de formar profesionales calificados en la enseñanza de prácticas integradoras y complementarias para su avance en el SUS. Por tanto, el objetivo de este estudio fue describir las percepciones sobre la homeopatía desde la perspectiva de estudiantes de cursos de salud en universidad privada de Recife-PE. Fue realizado un estudio transversal, descriptivo y cuantitativo basado en la aplicación de un cuestionario a 500 estudiantes de salud, entre octubre y noviembre de 2018. Aunque el $65,2 \%$ de los estudiantes informa haber oído hablar de la homeopatía, 73\% informó tener poco o ningún conocimiento sobre las formas de tratamiento. Además, el 82,8\% de los estudiantes manifestó no conocer la PNPIC, ni saber que los medicamentos homeopáticos son distribuidos gratuitamente por el SUS (86\%). Los estudiantes están interesados en incluir la homeopatía como disciplina en sus cursos, para poder ofrecer una atención integral a los pacientes. Existe la necesidad de que las instituciones de educación superior inviertan y promuevan disciplinas en homeopatía, formando profesionales con mayor conocimiento sobre PIC, lo que conducirá a una mayor información para la población, mejorando la salud individual y colectiva a través de la salud público.
\end{abstract}

Palabras clave: Educación superior, homeopatía, terapias alternativas, SUS.

\section{INTRODUÇÃo}

A homeopatia foi popularizada por Samuel Hahnemann no século XVIII, em seus livros "Organon da Arte de Curar" e "Tratado sobre as Doenças Crônicas”, nas quais apresentou, após anos de estudos e prática homeopática na clínica, seus princípios filosóficos e doutrinários. Desde então, tem se tornado uma prática medicinal alternativa popular e com filosofia própria, embora gere debates na comunidade científica (LOCH-NECKEL et al., 2010; NAGARE; USHIR, 2020). 
A homeopatia é baseada na administração de medicamentos que são preparados a partir de fontes animais, minerais, medicamentos sintéticos entre outros, que são diluídas em diferentes concentrações, da escala decimal, centesimal à milesimal (REHMAN; AHMAD, 2017). No modelo homeopático, a cura do paciente se dá pela similitude, com a administração de doses diluídas de substâncias para estimular reações homeostáticas curativas contra uma doença, induzindo o organismo a reagir contra os seus próprios distúrbios (TEIXEIRA, 2006).

O modelo de tratamento homeopático chegou ao Brasil há quase 200 anos, quando os primeiros consultórios e farmácias foram iniciados pelo médico francês Benoit-Mure, no Rio de Janeiro (CORRÊA, 2006). Porém, só a partir de 1980, quando a homeopatia foi reconhecida como especialidade médica pelo Conselho Federal de Medicina (CFM) a partir da Resolução CFM no 1.000/80, é que em algumas regiões do país, os usuários dos serviços de saúde passaram a ter acesso ao atendimento homeopático por médicos especialistas (LOCH-NECKEL et al., 2010).

Anos depois, devido ao aumento no número de farmácias homeopáticas sem profissionais qualificados, o Conselho Federal de Farmácia (CFF), na Resolução no 232 de 6 de maio de 1992 (revogada e posteriormente editada nas Resoluções nº 440 de 22 de setembro de 2005 e nº 576 de 28 de junho de 2013) também reconheceu a homeopatia como especialidade farmacêutica. Logo após, em 1995, os médicos veterinários também passaram a ter a especialidade reconhecida por seu conselho (OLIVEIRA, 2016). A partir do reconhecimento da homeopatia como especialidade médica, ela pôde ser inserida no Sistema Único de Saúde (SUS), sendo reconhecida técnico-cientificamente, popularmente e tradicionalmente (LACERDA, 2011).

Em 2006, o Ministério da Saúde do Brasil publicou a Política Nacional de Práticas Integrativas e Complementares (PNPIC) no Sistema Único de Saúde (SUS), por meio da Portaria n 971/2006, para atuação em todos os níveis de atenção à saúde, com intuito de beneficiar a população. Segundo esse documento, as Práticas Integrativas e Complementares (PICs) compreendem um sistema de abordagens sobre diversas práticas, tais como acupuntura, homeopatia e fitoterapia. Porém, estudos tem mostrado que as PICs não são conhecidas por grande parte dos estudantes de saúde, que também não sabem que os medicamentos homeopáticos são oferecidos gratuitamente pelo SUS (OLIVEIRA, 2014; SANTOS et al., 2018).

Com a publicação da PNPIC, aumentou a demanda por profissionais de saúde com formação ampla e qualificação em práticas integrativas e complementares para que as PICs pudessem avançar no SUS, sendo necessária a inserção dessas práticas em cursos de saúde. Nos últimos 15 anos, em Instituições de Ensino Superior (IES) públicas houve um aumento na oferta de disciplinas que contemplam as terapias complementares, evidenciando que tais práticas estão sendo mais buscadas 
em diferentes áreas, favorecendo sua abordagem de forma multidisciplinar nos serviços de saúde (NASCIMENTO, 2018).

No entanto, a falta de conhecimento sobre homeopatia que ainda há entre profissionais e estudantes de saúde tem permitido a visão simplista da área, que continua sendo associada equivocadamente apenas à efeito placebo, tratamento natural de aspecto místico-religioso, indicação apenas para doenças crônicas ou psicossomáticas, ausência de fundamento científico e não reconhecimento como especialidade médica (TEIXEIRA, 2004).

Visando a melhoria da saúde pública e construção do pensamento crítico, é importante que os estudantes recebam informações detalhadas sobre práticas integrativas. É preciso identificar a necessidade de uma possível inclusão de disciplinas que abordem homeopatia em cursos que tenham aptidão, beneficiando a formação do profissional de saúde generalista (AHMAD, 2014).

As discussões em torno de pesquisas envolvendo tratamento homeopático ainda são muitas, porém é necessário que seus desfechos sejam vistos e estudados nos cursos de saúde, para criar profissionais capacitados, críticos com formação científica e humanizados. Assim, torna-se necessário compreender o nível de conhecimento dos estudantes sobre homeopatia e apresenta-la como prática integrativa complementar prevista no SUS. O objetivo deste estudo foi descrever as percepções sobre homeopatia na perspectiva dos discentes da área de saúde de um centro universitário privado na cidade de Recife-PE.

\section{METODOLOGIA/ MATERIAL E MÉTODOS}

Foi realizado um estudo descritivo, quantitativo e do tipo transversal, entre os meses de outubro e novembro de 2018. A amostra foi constituída por 500 estudantes de diversos cursos de saúde de uma instituição de ensino superior privada situada em Recife, Pernambuco.

A amostra foi realizada por conveniência. Na IES estudada, na grade curricular dos cursos de saúde são oferecidas disciplinas importantes para a completa formação do profissional. Porém, a disciplina de homeopatia não é oferecida, obrigatoriamente ou optativamente.

Foram incluídos na amostra discentes regularmente matriculados nos seguintes cursos de saúde: Farmácia, Biomedicina, Enfermagem, Odontologia, Medicina Veterinária, Educação Física, Nutrição e Fisioterapia, do primeiro ao décimo período, com idade igual ou maior a 18 anos. Os participantes responderam a um questionário, no qual foram obtidos dados sociodemográficos (faixa etária, renda e sexo). 
Além disso, o questionário abordou questões de conhecimento prévio sobre homeopatia (definição, uso pessoal, forma do uso, efeitos colaterais, situações em que faria uso da terapia homeopática), conhecimento sobre as PNPIC, conhecimento sobre o fornecimento de homeopáticos pelo SUS, interesse em cursar homeopatia durante o curso, entre outras perguntas. Todos os dados obtidos foram tabulados em planilhas eletrônicas no Microsoft Excel, para obtenção dos resultados de frequência percentual de cada questão e variável estudada.

O projeto foi aprovado pelo Comitê de Ética e Pesquisa em Seres Humanos do Centro Universitário Maurício de Nassau (Protocolo nº 99973218.1.0000.5193). Participaram da pesquisa apenas os indivíduos que aceitaram responder o questionário, desde que concordassem em assinar o termo de consentimento livre e esclarecido (TCLE), no qual é garantido o sigilo das informações prestadas pelo participante. No TCLE está assegurado o compromisso ético de utilização das informações para efeito de pesquisa segundo a resolução 466/12 do Conselho Nacional de Saúde, respeitando o sigilo e a confiabilidade das informações.

\section{RESULTADOS}

\section{Perfil dos acadêmicos pesquisados}

Foram entrevistados 500 estudantes dos cursos de saúde da instituição, que responderam ao questionário integralmente. A maior parte da amostra foi composta por estudantes do sexo feminino (354/500), representando $70,8 \%$, enquanto $29,2 \%$ dos estudantes pertenceram ao sexo masculino (146/500). A média de idade dos estudantes foi de 25,9 anos e, em sua maioria, residiam em zona urbana (478/500), representando 95,6\% da amostra, enquanto apenas 4,4\% deles residiam em área rural (22/500). A renda familiar predominante (270/500) foi de 1 a 3 salários mínimos, que representou $54,0 \%$ das respostas.

\section{Percepções dos discentes acerca da homeopatia}

Quando questionados sobre ter ouvido falar, ter lido ou ter algum conhecimento sobre homeopatia, 326 estudantes $(65,2 \%)$ responderam que sim, enquanto $174(34,8 \%)$ responderam que não, conforme exibe a Tabela 1. A maioria dos estudantes do curso de farmácia (128/146) afirmaram que sim, representando $87,7 \%$ dos estudantes do curso que participaram da pesquisa. 0 curso de fisioterapia obteve o maior percentual de estudantes que não haviam ouvido falar/lido ou não possuem conhecimento sobre homeopatia, representando 58,8\% (34/48) dos discentes do curso. 
Tabela 1 - Distribuição dos estudantes, por curso, que já ouviram falar, leram ou tem conhecimento sobre homeopatia

\begin{tabular}{lcccccc}
\hline Cursos & \multicolumn{2}{c}{ Sim } & \multicolumn{2}{c}{ Não } & \multicolumn{2}{c}{ Total } \\
& $\mathbf{n}$ & $\mathbf{\%}$ & $\mathbf{n}$ & $\mathbf{\%}$ & $\mathbf{n}$ & $\mathbf{\%}$ \\
\hline Farmácia & 128 & 87,7 & 18 & 12,3 & 146 & 29,2 \\
Biomedicina & 41 & 61,2 & 26 & 38,8 & 67 & 13,4 \\
Enfermagem & 49 & 53,8 & 42 & 46,2 & 91 & 18,2 \\
Odontologia & 26 & 54,2 & 22 & 45,8 & 48 & 9,6 \\
Medicina veterinária & 22 & 55,0 & 18 & 45,0 & 40 & 8,0 \\
Educação física & 17 & 51,5 & 16 & 48,5 & 33 & 6,6 \\
Nutrição & 29 & 70,7 & 12 & 29,3 & 41 & 8,2 \\
Fisioterapia & 14 & 41,2 & 20 & 58,8 & 34 & 6,8 \\
\hline Total & $\mathbf{3 2 6}$ & $\mathbf{6 5 , 2}$ & $\mathbf{1 7 4}$ & $\mathbf{3 4 , 8}$ & $\mathbf{5 0 0}$ & $\mathbf{1 0 0}$ \\
\hline
\end{tabular}

Fonte: Própria.

Sobre a fonte do conhecimento sobre homeopatia, como apresenta a Tabela 2, parte dos estudantes que conheciam (147/500), representando $29,4 \%$, relataram que foi na faculdade que obtiveram o conhecimento. Outros $27 \%$ responderam internet (104/500), enquanto 9,4\% (47/500) responderam "outros", entre os quais citaram livros, artigos, jornais, televisão, congressos da instituição, estágios na área, trabalho, farmácia comunitária e de manipulação, através de amigos, familiares e consultas com médicos naturalistas e psicólogos. Revistas também foram citadas por apenas 26 estudantes, representando 5,2\% da amostra. Apesar da maioria dos estudantes $(59,4 \%)$ não ter ouvido falar da homeopatia em nenhuma disciplina, as disciplinas apresentadas como fonte do conhecimento sobre o tema mais citadas foram farmacognosia (21\%), farmacotécnica (3,2\%) e tecnologia dos medicamentos $(2,8 \%)$.

Uma parte da amostra (13,6\%), composta por 68 dos 500 estudantes, respondeu "Outras disciplinas", citando entre elas farmacologia, síntese e planejamento de fármacos, farmácia hospitalar, patologia, fisiologia, farmacologia veterinária, terapêutica medicamentosa, farmacoterapia, semiologia, estágio supervisionado, bioquímica, biologia molecular, dietoterapia, hematologia e biotecnologia. 
Tabela 2 - Fonte de conhecimentos sobre homeopatia

\begin{tabular}{lcc}
\hline Fonte de conhecimento sobre homeopatia & $\mathbf{n}$ & $\mathbf{\%}$ \\
147 & 29,4 \\
Faculdade & 104 & 20,8 \\
Internet & 47 & 9,4 \\
Outros & 26 & 5,2 \\
Revistas & 176 & 35,2 \\
Não conhece & & \\
Conhecimento sobre o Tema em Alguma Disciplina da Graduação & 105 & 21,0 \\
Farmacognosia & 16 & 3,2 \\
Farmacotécnica & 14 & 2,8 \\
Tecnologia dos medicamentos & 68 & 13,6 \\
Outras disciplinas & 297 & 59,4 \\
Nenhuma & $\mathbf{5 0 0}$ & $\mathbf{1 0 0}$ \\
\hline Total & & \\
\hline
\end{tabular}

Fonte: Própria.

\section{Conhecimento dos estudantes sobre o modelo de tratamento homeopático}

Sobre a percepção de formas do tratamento homeopático, como exibido na Tabela 3, a maioria dos estudantes (219/500), representando 43,8\%, afirmou não saber qual o modelo, enquanto apenas $14,8 \%$ (74/500) dos participantes relataram que o tratamento é relacionado ao uso de glóbulos ou gotas.

Autoavaliando-se sobre o nível de conhecimento em homeopatia, 73\% dos estudantes (365/500) declararam ter pouco ou nenhum conhecimento, 26,4\% (132/500) afirmaram ter médio ou razoável conhecimento e apenas $0,6 \%$ discentes (3/500) responderam ter amplo conhecimento sobre o tema. Logo após, foram abordadas questões sobre o uso de medicamentos homeopáticos e apenas $22,8 \%(114 / 500)$ já fizeram uso, enquanto $77,2 \%$ (386/500) responderam que não.

Dos estudantes que fizeram uso do tratamento homeopático, embora dois estudantes não tenham respondido, o percentual de 45,6\% (52/114) relatou ter sido por automedicação, enquanto $32,5 \%$ (37/114) afirmou que foi por indicação de alguém e 20,2\% (23/114) responderam que o tratamento foi indicado por receituário médico. Sobre a presença de efeitos colaterais, apenas 3,5\% 
(4/114) responderam que sim, enquanto 91,2\% (104/114) responderam que não. Seis estudantes não responderam quando questionados sobre a presença de efeitos colaterais.

Tabela 3. Percepção sobre formas de tratamento homeopático

\begin{tabular}{lcc}
\hline Percepção & $\mathbf{n}$ & $\mathbf{\%}$ \\
\hline Uso de ervas & 161 & 32,2 \\
Uso de chás & 18 & 3,6 \\
Uso de glóbulos ou gotas & 74 & 14,8 \\
Uso de florais & 28 & 5,6 \\
Não sei & 219 & 43,8 \\
\hline Total & $\mathbf{5 0 0}$ & $\mathbf{1 0 0}$ \\
\hline
\end{tabular}

Fonte: Própria.

Em relação as situações em que fariam o uso da terapia homeopática, 38,2\% (191/500) responderam que utilizariam em casos de ansiedade, enquanto 22,6\% (113/500) citaram casos de dor e inflamação, 8,4\% (42/500) relataram que utilizariam em doenças graves e 6\% (30/500) responderam outros, mencionando o uso sob orientação médica em casos de asma, virose, gripes e resfriados, gordura no fígado, tratamento de cistos, melhora do retorno venoso, má digestão e colesterol, infecção urinária, insônia/depressão, queda de cabelo e tosse produtiva. Um total de 124 estudantes $(24,8 \%)$ não respondeu esta questão.

\section{o Sistema Único de Saúde e a homeopatia}

Mediante à informação de que a homeopatia está incluída no SUS foi perguntado se os discentes conheciam as PNPIC. Apenas 17,2\% (86/500) afirmaram conhecer, enquanto 82,8\% (414/500) relataram desconhecer. A respeito dos medicamentos homeopáticos serem oferecidos de forma gratuita pelo SUS, apenas 14,0\% (70/500) sabiam dessa informação e 86,0\% (430/500) afirmaram não saber.

Diante da ausência da disciplina nos cursos de saúde, foi perguntado se gostariam que a disciplina "homeopatia" fosse oferecida na grade curricular do curso do participante da pesquisa e $52,6 \%(263 / 500)$ afirmaram que gostariam, mas como disciplina optativa/eletiva, enquanto $38,4 \%$ 
(192/500) gostariam de cursá-la em caráter obrigatório e 9,0\% (45/500) não demonstraram interesse em cursar.

Entre os cursos foi observado o nível de interesse em cursar esta disciplina (Tabela 4). Os estudantes de farmácia foram os que demonstraram maior interesse em cursar homeopatia, em caráter obrigatório (60,3\%) ou eletivo (36,3\%), totalizando 96,6\% (141/146) dos participantes do curso. O interesse em cursar a disciplina, somando-se o caráter obrigatório com o eletivo, seguiu nos cursos de enfermagem (95,6\%), nutrição (92,7\%), medicina veterinária (90\%), biomedicina (88,1\%), fisioterapia $(85,3 \%)$, odontologia $(81,2 \%)$ e educação física (78,8\%).

Tabela 4 - Interesse em cursar a disciplina de homeopatia nos cursos de graduação em saúde.

\begin{tabular}{lcccccc}
\hline \multirow{2}{*}{ Curso } & \multicolumn{2}{c}{ Sim, obrigatória } & \multicolumn{2}{c}{ Sim, optativa/eletiva } & \multicolumn{2}{c}{ Não } \\
& $\mathbf{n}$ & $\mathbf{\%}$ & $\mathbf{N}$ & $\mathbf{\%}$ & $\mathbf{n}$ & $\%$ \\
\hline Farmácia & 88 & 60,3 & 53 & 36,3 & 5 & 3,4 \\
Biomedicina & 20 & 29,9 & 39 & 58,2 & 8 & 11,9 \\
Enfermagem & 38 & 41,8 & 49 & 53,8 & 4 & 4,4 \\
Odontologia & 5 & 10,4 & 34 & 70,8 & 9 & 18,8 \\
Medicina veterinária & 14 & 35,0 & 22 & 55,0 & 4 & 10 \\
Educação física & 6 & 18,2 & 20 & 60,6 & 7 & 21,2 \\
Nutrição & 15 & 36,6 & 23 & 56,1 & 3 & 7,3 \\
Fisioterapia & 6 & 17,7 & 23 & 67,6 & 5 & 14,7 \\
\hline Total & $\mathbf{1 9 2}$ & & $\mathbf{2 6 3}$ & & $\mathbf{4 5}$ & \\
\hline
\end{tabular}

Fonte: Própria.

\section{DISCUSSÃO}

A falta de conhecimento sobre as Terapias Integrativas e Complementares no SUS pelos profissionais da saúde, que muitas vezes não conhecem a PNPIC, junto à ausência de disciplinas que abordem esses temas nos cursos de graduação em saúde, podem estar associadas ao baixo uso das PICs no Brasil (SOUZA et al., 2019). Apesar da maior parte dos estudantes participantes do presente estudo afirmarem ter algum tipo de conhecimento sobre homeopatia, principalmente entre os do curso de farmácia, vários estudos tem apresentado resultados divergentes e afirmam que o conhecimento da área por parte dos discentes é defasado, havendo pouca compreensão sobre a importância do tema (FREITAS; ALMEIDA, 2017).

Os estudantes de farmácia apresentaram o maior conhecimento sobre homeopatia provavelmente por, durante o curso, aprenderem mais sobre dispensação de medicamentos e orientação de uso (ISRAEL, 2016). 
Além disso, por possuírem mais disciplinas nas áreas de produção e tecnologia de medicamentos e farmacognosia, é esperado que o conhecimento desses seja maior ou que tenham ouvido menção à homeopatia em algum momento do curso.

De um modo geral os discentes da área de saúde já ouviram falar sobre homeopatia em algum momento de sua vida acadêmica (GAVIN, 2011). Ainda assim, os resultados demonstram que esta prática ainda não está totalmente elucidada para os discentes, ou seja, faltam informações concretas para os futuros profissionais de saúde, consumidores e alguns futuros prescritores (BALLESTEROSPEÑA, 2015).

Apesar de ser reconhecida como especialidade médica, no presente estudo não houve participantes do curso de medicina, em virtude dos estudantes se localizarem em outro campus da instituição. Ainda assim, ao longo dos anos, a homeopatia vem sendo incorporada como disciplina em cursos médicos de IES públicas, como Universidade Federal de Pernambuco, Universidade Federal da Paraíba, Universidade Federal Fluminense entre outras (OLIVEIRA et al., 2018).

Pequena parte dos discentes relacionou corretamente a percepção da homeopatia com glóbulos ou gotas, enquanto uma grande parte relacionou erroneamente ao uso de ervas. Isso demontra que há equívoco entre as noções de homeopatia e fitoterapia. O estudo de Amorim e colaboradores (2020), realizado com mais de 800 participantes, também encontrou compreensão equivocada em relação à homeopatia, com muitos afirmando existir princípios ativos em medicamentos homeopáticos, além de pouco domínio sobre fitoterapia. Estes relatos indicam que, embora as PICs estejam sendo mencionadas, é necessário que elas sejam oferecidas nas grades curriculares, necessitando que o conteúdo não seja apenas apresentado de maneira paradigmática, mas ressaltando a integração das PIC no cuidado integral ao indivíduo (NASCIMENTO et al., 2018; TESSER et al., 2018).

A autoavaliação do conhecimento sobre homeopatia revela pouco ou nenhum conhecimento por parte dos discentes. No entanto, as instituições de ensino devem considerar que mesmo que alguns estudantes não tenham interesse em trabalhar nesta especialidade, eles deveriam ter acesso ao conhecimento para poder optar e entender a prática para um tratamento integral (LOCH-NECKEL, 2010).

Assim como o presente estudo, diversos outros tem encontrado baixos percentuais de utilização de terapia homeopática, diferente de pesquisas em outros países, como Bangladesh, onde mais da metade dos estudantes entrevistados relataram o uso de algum medicamento homeopático (SAHA, 2010). Em Bangladesh, no ano de 2009, existia uma disponibilidade de aproximadamente 33 homeopatas para cada 10.000 habitantes e, nos anos seguintes, vários médicos homeopatas foram 
nomeados para o Projeto de Desenvolvimento do Setor de Saúde, População e Nutrição, vinculado ao Ministério da Saúde e Bem Estar Familiar do país, com muitos pacientes buscando o serviço (AHMED et al., 2009; AHMED et al., 2015). A falta de conhecimento sobre homeopatia por parte dos profissionais possivelmente está associada à ausência de estímulo ao seu uso (THIAGO, 2011).

Outros estudos também tem relatado a utilização de medicamentos homeopáticos por automedicação ou indicação de alguém, a exemplo de um estudo conduzido na Coreia do Sul, que relatou familiares e amigos como a principal fonte de indicação para uso de medicina complementar e alternativa (OCK, 2009).

Embora a homeopatia esteja incluída no SUS e a PNPIC forneça uma série de abordagens sobre essa e outras práticas, a maioria dos discentes não está ciente disso, além de não saber que o SUS oferece medicamentos homeopáticos de forma gratuita. Em pesquisa recente realizada com profissionais experientes, atuantes em unidades de Saúde da Família em Santa Catarina, também pôde ser observado o desconhecimento sobre PNPIC (MATTOS, 2018).

Em geral, os estudantes mostraram interesse em cursar a disciplina de homeopatia nos cursos de graduação em saúde, provavelmente na busca de aprofundar o conhecimento no tema. Em pesquisa realizada em IES públicas do Rio de Janeiro, sobre a oferta de disciplinas em Práticas Integrativas e Colaborativas, a disciplina de homeopatia, junto à meditação e práticas corporais, foram as mais frequentes, porém em perfil opcional e informativo, ressaltando a importância de ofertá-las de maneira integrada para possibilitar também uma formação mais prática (NASCIMENTO, 2018).

Outros cursos também demonstraram interesse em cursar uma disciplina de homeopatia. Estudantes de fisioterapia, por exemplo, apesar de aproximarem-se com técnicas de acupuntura e quiropraxia, que são métodos complementares inseridos na PNPIC junto à homeopatia e fitoterapia (BALLESTEROS-PEÑA, 2017), em sua maioria, declararam não ter ouvido falar da homeopatia, embora tenham demonstrado interesse em cursar a disciplina durante a graduação.

Apesar dos poucos estudos relacionados à homeopatia em odontologia, sua utilização clínica tem aumentado e é necessário realizar mais relatos de caso e estudos nessa área (COSTA et al., 2016). É possível observar a eficácia de métodos alternativos em procedimentos odontológicos com ou sem associação da terapia tradicional alopática, através de profissional habilitado (ALMEIDA, 2006). Chebel (2013), por exemplo, em sua tese, verificou que o tratamento homeopático em pacientes com síndrome de ardência bucal reduziu os sintomas em mais de $44 \%$ do grupo que recebeu o tratamento homeopático, enquanto apenas cerca de $12 \%$ dos que utilizaram um placebo apresentaram a redução. 
Ainda assim, pode ser observado que existem profissionais de saúde que atuam na área e muitos discentes não sabem de suas futuras competências.

$\mathrm{Na}$ área da medicina veterinária, a homeopatia tem sido utilizada no controle da diarreia em suínos, no tratamento e bem estar de animais selvagens, no controle das enteroparasitoses, na produção de leite entre outros (BONAMIN, 2019). Estudo realizado em felinos com hipertireoidismo, inclusive demonstrou que através do tratamento homeopático os sinais e sintomas apresentados foram normalizados (CATONOCE, 2017).

Os discentes de farmácia, embora tenham demonstrado o maior interesse em cursar uma disciplina sobre homeopatia na grade curricular do curso, não foram os únicos. Estudo em Serra Leoa destacou a preferência dos estudantes pelo aprendizado em medicina complementar e alternativa e a necessidade de sua inclusão nos currículos (JAMES, 2014). Um estudo na Austrália também evidenciou que é preciso acesso aos recursos medicina complementar e alternativa, bem como do ensino desses métodos e terapias (NAIDU, 2005).

\section{CONSIDERAÇõES FINAIS}

Entre os estudantes de cursos de saúde de um centro universitário de Recife, Pernambuco ainda não está claro o conceito de homeopatia e muitos nunca ouviram falar, além de considerarem ter pouco ou nenhum conhecimento a respeito. Entretanto, com exceção dos estudantes de farmácia, cuja maioria apresentou interesse em cursar uma disciplina de homeopatia de forma obrigatória na grade curricular, a maior parte dos estudantes participantes de outros cursos, também possuem interesse em cursar a disciplina, porém como eletiva/optativa. Discentes de farmácia obtiveram destaque nos conhecimentos específicos sobre medicamentos homeopáticos, porém assim como os de outros cursos, em sua maioria alegaram ter pouco ou nenhum conhecimento no tema.

Investimentos são necessários no ensino das práticas integrativas e complementares por parte das instituições de ensino superior, oferecendo esta disciplina ao menos em caráter optativa/eletiva, pois a inserção da homeopatia na grade curricular desses cursos fornecerá informações sobre outras modalidades terapêuticas pelos estudantes, ampliando a atuação desses futuros profissionais de modo a beneficiar as necessidades gerais de saúde de toda população, afinal é um modelo de tratamento que, embora ainda muito discutido, está integrado ao Sistema Único de Saúde, de forma gratuita. 
Percepções sobre homeopatia na perspectiva de discentes dos

cursos de saúde de um centro universitário de Recife-PE

\section{REFERÊNCIAS}

AHMAD, A.; KHAN, M.V.; BANDARI, D.K.; KUMAR, G.S.; RODRIGUEZ, S.P.; PATEL, I.B. Beliefs, attitudesand self-use of Ayurveda, Yoga and Naturopathy, Unani, Siddha, and Homeopathy medicines among senior pharmacy students: An Exploratory insight from Andhra Pradesh, India. Pharmacognosy Research. v.7, n.4, p.302-308, 2014 . Disponível em <https://www.ncbi.nlm.nih.gov/pmc/articles/PMC4660507>. Acesso em 10 de agosto de 2018.

AHMED, S.M.; ALAM, B. B.; ANWAR, I.; BEGUM, T.; HUQUE, R.; KHAN, J.A.M.; NABABAN, H.; OSMAN, F.A. Bangladesh health system review. Health System in Transition, v.5, n.3, 2015. Disponível em:< https://apps.who.int/iris/bitstream/handle/10665/208214/9789290617051_eng.pdf?sequence=1\&isAllowed=y >. Acesso em 22 de novembro de 2020.

AHMED, S.M.; HOUSSAIN, A.; CHOWDHURY, M.R. Informal sector providers in Bangladesh: how equipped are they to provide rational health care?. Health Policy and Planning, v.24, n.6, p.467-478, 2009. Disponível em < https://academic.oup.com/heapol/article/24/6/467/913391 >. Acesso em 22 de novembro de 2020.

ALMEIDA, A. D.; WERKMAN, C.; CANETTIERI, A. C. V. Uso de terapias alternativas no consultório odontológico: uma revisão da literatura. In: ENCONTRO LATINO-AMERICANO DE INICIAÇÃO CIENTÍFICA, 10.; ENCONTRO LATINO-AMERICANO DE PÓSGRADUAÇÃO, 6., 2006, São José dos Campos. Anais [...]. São José dos Campos, 2006. Disponível em < http://www.inicepg.univap.br/cd/INIC_2006/inic/inic/03/INIC0000948.pdf>. Acesso em 02 de setembro de 2019.

AMORIM, L.S. BRANDÃO, H.N.; FERREIRA, L.F.; FIGUEREDO, C.A.; SOUSA, M.S.; COSTA, D.A. Conhecimento sobre Homeopatia e Fitoterapia em comunidade universitária. Revista Brasileira de Educação e Saúde, v.10, n.3, p.128-135, 2020. Disponível em < https://gvaa.com.br/revista/index.php/REBES/article/view/7927/0 >. Acesso em 3 de novembro de 2020.

BALLESTEROS-PEÑA, S.; FERNÁNDEZ-AEDO, I. Conocimientos y actitudes sobre terapias alternativas y complementarias enestudiantes de ciencias de la salud. Investigación en Educación Médica.v.4, n.16, p. 207-215, 2015. Disponível em <https://www.sciencedirect.com/science/article/pii/S2007505715000253 >. Acesso em 30 de julho de 2018.

BIANCHI, M.; MENEGÓCIO, A.M.; BRUZADELLI, R.; SATIE ABE, K. Atuação do Enfermeiro na Terapia Alternativa: Homeopatia. Ensaios e Ciência: Ciências Biológicas, Agrárias e da Saúde, v.19 n.1, p.42-46, 2015. Disponível em < https://revista.pgsskroton.com/index.php/ensaioeciencia/article/view/3015\#: :text=A\%20homeopatia\%20vem\%20ganha ndo\%20relev\%C3\%A2ncia,e\%20promo\%C3\%A7\%C3\%A30\%20de\%20sua\%20sa\%C3\%BAde. >. Acesso em 21 de março de 2018.

BONAMIN, L.V. A pesquisa básica sobre homeopatia no Brasil. Revista de Homeopatia, v.82, n.3/4, p.48-49, 2019. Disponível em: < http://revista.aph.org.br/index.php/aph/article/download/460/558 >. Acesso em 5 de janeiro de 2021.

BRASIL. Ministério da Saúde. Portaria no 971, de 3 de maio de 2006. Aprova a Política Nacional de Práticas Integrativas e Complementares (PNPIC) no Sistema Único de Saúde. Brasília, 2006. Diário Oficial da República Federativa do Brasil. Brasília,

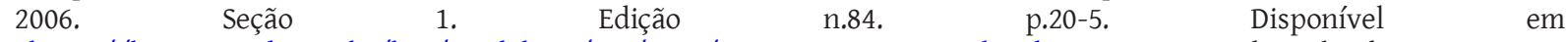
<https://bvsms.saude.gov.br/bvs/saudelegis/gm/2006/prt0971_03_05_2006.html>. Acesso em 10 de junho de 2019.

CATONOCE, E. Homeopatia e hipertireoidismo felino. 2017. 104 f. Monografia (Especialização em Homeopatia) - Centro Alpha de Ensino Associação Paulista de Homeopatia, São Paulo, 2017. Disponível em <https://pesquisa.bvsalud.org/portal/resource/pt/hom-11866?lang=en>. Acesso em 10 de junho de 2019.

CFF. Conselho Federal de Farmácia. Resolução no 440, de 22 set. 2005. Dá nova redação à Resolução nº 335/98 do Conselho Federal de Farmácia, que dispõe sobre as prerrogativas para o exercício da responsabilidade técnica em homeopatia. Diário Oficial [da República Federativa do Brasil]. Brasília, DF, n.206, p.147, 26 out. 2005. Seção 1. Disponível em < https://www.cff.org.br/userfiles/file/resolucoes/440.pdf>. Acesso em 8 de junho de 2019.

CHEBEL, I. F. O. Ação do tratamento homeopático na sintomatologia da síndrome da ardência bucal em duas fases: estudo duplo cego placebo controlado e estudo aberto. 2013. Tese (Doutorado em Diagnóstico Bucal). Universidade de São Paulo, São Paulo, 2013.

CORREA, A. D.; SIQUEIRA-BATISTA, R.; QUINTAS, L.E.M; SIQUEIRA-BATISTA, R. Similia Similibus Curentur: revisitando aspectos históricos da homeopatia nove anos depois. História, Ciências, Saúde-Manguinhos, Rio de Janeiro, v. 13, n. 1, p. 13-31, Mar. 2006. Disponível em < https://www.scielo.br/scielo.php?script=sci_arttext\&pid=S010459702006000100002\&lng=pt\&tlng=pt >. Acesso em 8 de junho de 2020 .

COSTA, R.F; VOLPATO, S.; GALLON, A.; DIRSCNABEL, A.J. BIOCAMPO ENERGÉTICO: ATUAÇÃO DA HOMEOPATIA NA ODONTOLOGIA. Unoesc e Ciência, v.7, n.2, p.137-144, 2016. Disponível em: < https://core.ac.uk/download/pdf/235124312.pdf >. Acesso em 2 de dezembro de 2020. 
FREITAS, L. F.; ALMEIDA, A.F.S. Conhecimento sobre a homeopatia entre os acadêmicos da área da saúde da Faculdade Ciências da Vida. Revista Brasileira de Ciências da Vida, v.5, n.5, 2017. Disponível em < http://jornalold.faculdadecienciasdavida.com.br/index.php/RBCV/article/view/388 >. Acesso em 22 de novembro de 2020.

GAVIN, R. O. S.; OLIVEIRA, M. H. P. DE; GHERARDI-DONA, E. C. DA S. Terapias alternativas complementares: uma visão do conhecimento dos acadêmicos de enfermagem. Ciência, Cuidado e Saúde, v. 9, n. 4, p. 760-765, jul. 2011. Disponível em < http://periodicos.uem.br/ojs/index.php/CiencCuidSaude/article/view/13827 >. Acesso em 18 de julho de 2020.

ISRAEL. A.L.M. Atenção, dispensação e prescrição farmacêuticas em homeopatia. 2016. Monografia- (Especialização em Homeopatia). Centro Alpha de Ensino Associação Paulista de Homeopatia, São Paulo, 2016. Disponível em < https://pesquisa.bvsalud.org/portal/resource/pt/hom-11136 >. Acesso em 20 de agosto de 2019.

JAMES, P. B.; BAH, A. J. Awareness, use, attitude and perceived need for Complementary and Alternative Medicine (CAM) education among undergraduate pharmacy students in Sierra Leone: a descriptive cross-sectional survey. BMC complementary and alternative medicine, v.14, p. 438. 2014. Disponível em < https://www.ncbi.nlm.nih.gov/pmc/articles/PMC4236455/ >. Acesso em 8 de julho de 2020.

LACERDA, A. Homeopatia popular e praticada por médicos: desafios da integração das práticas no contexto da promoção da saúde. In: PINHEIRO, R.; JUNIOR, S.A.G. Cidadania no cuidado: o universal e o comum na integralidade das ações em saúde. Rio de Janeiro: IMS/Uerj-Cepesc. 2011. p. 305 - 317.

LOCH-NECKEL, G.; CARMIGNAN, F.; CREPALDI, M. A. A homeopatia no SUS na perspectiva de estudantes da área da saúde. Rev. bras. educ. med., Rio de Janeiro, v.34, n. 1, p. 82-90, Mar. 2010. Disponível em < http://www.scielo.br/scielo.php?script=sci_arttext\&pid=S0100-55022010000100010\&lng=en\&nrm=iso>. Acesso em $8 \mathrm{de}$ junho de 2020.

MAHMOUDIAN, A.; SADRI, G. Homeopathy satisfaction in Iran. Iranian Journal of Nursing And Midwifery Research. v.19, n.5, p. 496-502, 2014. Disponível em < https://www.ncbi.nlm.nih.gov/pmc/articles/PMC4223967/ >. Acesso em 15 de janeiro de 2020 .

MARQUES, L.A.M.; VALE, F.V.V.R.; NOGUEIRA, V.A.S.; MIALHE, F.L.; SILVA, L.C. Atenção farmacêutica e práticas integrativas e complementares no SUS: Conhecimento e aceitação por parte da população São Joanense. Physis - Revista de Saúde Coletiva. Rio de Janeiro, v. 21, n.2, p. 663-674, 2011. Disponível em < https://www.scielo.br/scielo.php?script=sci_arttext\&pid=S0103-73312011000200017\&lang=en >. Acesso em 18 de janeiro de 2020.

MATTOS, G.; CAMARGO, A.; SOUSA, C. A.; ZENI, A. L. B. Plantas medicinais e fitoterápicos na Atenção Primária à Saúde: a percepção dos profissionais. Ciência \& Saúde Coletiva, v.23, n.11, p.3735-3744, 2018. Disponível em < https://www.scielo.br/scielo.php?script=sci_abstract\&pid=S1413-81232018001103735\&lng=pt\&nrm=iso >. Acesso em 20 de junho de 2020.

NAGARE, B.D.; USHIR, Y. V. Homeopathic Pharmacy's Legislation in India- A Review. British Journal of Medical and Health Research, v.7, n. 6, 2020. Disponível em < http://bjmhr.com/bjmhr_admin/uploads/publish_pdf/BJMHR\%20806006.pdf $>$. Acesso em 20 de dezembro de 2020.

NAIDU, S.; WILKINSON, J. M.; SIMPSON, M.D. Attitudes of Australian Pharmacists Toward Complementary and Alternative Medicines. Ann Pharmacother, v.39, n.9, p.1456-1461, 2005. Disponível em < https://journals.sagepub.com/doi/abs/10.1345/aph.1G089 >. Acesso em 13 de agosto de 2020.

NASCIMENTO, M.C.; ROMANO, V.F.; CHAZAN, A.C.S.; QUARESMA, C.H. Formação em práticas integrativas e complementares em saúde: desafios para universidades públicas. Trabalho Educação Saúde, Rio de Janeiro, v.16, n.2, p. 751-772, 2018. Disponível em < https://www.scielo.br/scielo.php?pid=S1981-77462018000200751\&script=sci_abstract >. Acesso em $18 \mathrm{de}$ junho de 2020.

OCK S.M.; CHOI, J.Y.; CHA, Y.S.; LEE J.; CHUN, M.S.; HUH. C.H.; LEE, S.Y.; LEE, S.J. The use of Complementary and Alternative Medicine in a General Population in South Korea: Results from a National Survey in 2006. Jornal of Korean Medical Science. v.24, n.1, p.1-6, 2009. Disponível em < https://www.ncbi.nlm.nih.gov/pmc/articles/PMC2650987/ >. Acesso em 8 de setembro de 2020 .

OLIVEIRA, A.A.; SALVI, J.O. Percepções de acadêmicos de farmácia sobre a homeopatia. Revista de Homeopatia. São Paulo, v. 77(1/2): p.16-20, 2014. Disponível em < http://revista.aph.org.br/index.php/aph/article/view/276/339 >. Acesso em 9 de junho de 2020. 
Percepções sobre homeopatia na perspectiva de discentes dos cursos de saúde de um centro universitário de Recife-PE

OLIVEIRA, I.F.; PELUSO, B.H.B.; FREITAS, F.A.C.; NASCIMENTO, M.C. Homeopatia na Graduação Médica: Trajetória da Universidade Federal Fluminense. Revista Brasileira de Educação Médica, v.42, n.1, p.92-102, 2018. Disponível em < https://www.scielo.br/pdf/rbem/v42n1/0100-5502-rbem-42-01-0094.pdf >. Acesso em 3 de novembro de 2020.

OLIVEIRA, L.M. Ação da calendula officinalis 6 ch e spray de quitosana na cicatrização de feridas cutâneas em ratas diabéticas. 2016. 37 f. Dissertação (Mestrado) - Escola de Veterinária e Zootecnia, Programa de Pós-graduação em Ciência Animal, Universidade Federal de Goiás, Goiânia, 2016. Disponível em < https://repositorio.bc.ufg.br/tede/handle/tede/6368\#: :text=TEDE\%3A\%20A\%C3\%A7\%C3\%A30\%20da\%20calendula\%20offi cinalis,feridas\%20cut\%C3\%A2neas\%20em\%20ratas\%20diab\%C3\%A9ticas\&text=Resumo\%3A,dificuldade\%20de\%20cicatriza\% C3\%A7\%C3\%A30\%20de\%20feridas. >. Acesso em 27 de março de 2019.

REHMAN, T; AHMAD, S. Introduction of homeopathy and homeopathic medicines: A review. International Journal of Homoeopathic Sciences, v.1, n.1, p.21-25, 2017. Disponível em < https://www.homoeopathicjournal.com/articles/7/1-1-8696.pdf $>$. Acesso em 18 de dezembro de 2020.

SAHA. B.L.; SEAM. M.R.S.; ISLAM, M.M.; DAS, A.; AHAMED, S.K.; KARMAKAR, P.; ISLAM, M.F.; KUNDU, S.K. General perception and self-practice of complementary and alternative medicine (CAM) among undergraduate pharmacy students of Bangladesh. BMC Complementary and Alternative Medicine. v.17, n.1, p. 314, 2017. Disponível em < https://pubmed.ncbi.nlm.nih.gov/28615021/ >. Acesso em 21 de maio de 2019.

SANTOS, L.L.; MIRANDA, S. P.; CLEMENTE, V. C.; NOGUEIRA, M.C. Conhecimento e aceitação das práticas integrativas e complementares por estudantes de medicina. Revista de APS, n. 21, v.4, p. 646-666, 2018. Disponível em < https://periodicos.ufjf.br/index.php/aps/article/view/16546/20131 >. Acesso em 12 de novembro de 2020.

SOUZA, G.H.C.; LEITE, R. S.; YOSHIDA, E. H.; SANTOS, N. S. USO DA HOMEOPATIA NO SISTEMA ÚNICO DE SAÚDE. Revista Saúde em Foco, n. 11, p.346-355, 2019. Disponível em < https://portal.unisepe.com.br/unifia/wpcontent/uploads/sites/10001/2019/03/027_USO-DA-HOMEOPATIA-NO-SISTEMA-\%C3\%9ANICO-DE-SA\%C3\%9ADE.pdf >. Acesso em 22 de novembro de 2020.

TEIXEIRA, M. Z. Homeopatia: ciência, filosofia e arte de curar. Revista de Medicina, v. 85, n. 2, p. 30-43, 21 jun. 2006. Disponível em < http://www.periodicos.usp.br/revistadc/article/view/59211 >. Acesso em 10 de abril de 2019.

TEIXEIRA, M.Z. Homeopatia, desinformação e preconceito. In: Anais do VIII Encontro Internacional de Pesquisas Institucionais em Homeopatia; 2004, São Paulo, Brasil.

TEIXEIRA, M.Z. Panorama mundial da educação médica em terapêuticas não convencionais (Homeopatia e Acupuntura). Revista de Homeopatia. São Paulo, v.80 (1/2): p. 18-39, 2017. Disponível em http://revista.aph.org.br/index.php/aph/article/view/392 >. Acesso em 10 de abril de 2019.

TEIXEIRA. M.Z:; LIN. C.A. Educação médica em terapias não convencionais. Revista de Medicina. São Paulo, v.92, n.4, p. 224235, 2013. Disponível em < http://www.revistas.usp.br/revistadc/article/view/80004 >. Acesso em 19 de maio de 2019.

TESSER, C. D.; SOUSA, I.M.C.; NASCIMENTO, M.C. Práticas Integrativas e Complementares na Atenção Primária à Saúde brasileira. Saúde Debate, v. 42, p. 174-188, 2018. Disponível em < https://www.scielo.br/scielo.php?script=sci_abstract\&pid=S0103-11042018000500174\&lng=en\&nrm=iso\&tlng=pt >. Acesso em 28 de novembro de 2020.

THIAGO, S.C.S.; TESSER, C.D. Percepção de médicos e enfermeiros da Estratégia de Saúde da Família sobre terapias complementares. Rev. Saúde Pública. São Paulo, v.45, n.2, p. 249-257, abr. 2011. Disponível em < https://www.scielo.br/scielo.php?pid=S0034-89102011000200003\&script=sci_abstract\&tlng=pt >. Acesso em 18 de maio de 2020.

\section{(c)) Br}

Este trabalho está licenciado com uma Licença Creative Commons - Atribuição 4.0 Internacional. 